\title{
Size Restrictions on the Passage of Overwintering Halyomorpha halys (Hemiptera: Pentatomidae) Through Openings
}

\author{
Benjamin D. Chambers, ${ }^{1,4, \infty}$ Thomas P. Kuhar, ${ }^{2, \bullet}$ Georg Reichard, ${ }^{1}$ Tracy C. Leskey, ${ }^{3}$ and \\ Annie R. Pearce ${ }^{1}$
}

\begin{abstract}
'Department of Building Construction, Virginia Tech, Bishop Favrao Hall, Room 430, 1345 Perry Street, Blacksburg, VA 24061, 2Department of Entomology, Virginia Tech, Price Hall, Room 216A, 170 Drillfield Drive, Blacksburg, VA 24061, ${ }^{3}$ US Department of Agriculture, Appalachian Fruit Research Station, 2217 Wiltshire Road, Kearneysville, WV 25430, and ${ }^{4}$ Corresponding author, e-mail: bdc0112@vt.edu
\end{abstract}

Subject Editor: Michael Rust

Received 20 September 2018; Editorial decision 7 January 2019

\begin{abstract}
Intentional and unintentional openings in a building's envelope provide opportunities for unwanted pests to enter buildings. The brown marmorated stink bug, Halyomorpha halys (Stål), is one such pest, causing a significant domestic winter nuisance in many locations. One important means of pest control is exclusion, or blocking openings through which they can enter, although some openings are intentional and cannot be completely blocked without putting a building at risk. To help understand what size openings are relevant to entry, adult $H$. halys ready for overwintering were driven out of heated boxes through openings designed to limit passage by their lateral pronotal and dorsoventral dimensions. Pronotally limited holes of $8 \mathrm{~mm}$ wide were passed by only one female ( $3.3 \%$ of those tested), and no females and only one male $(3.3 \%)$ passed through $7-\mathrm{mm}$-wide holes. For dorsoventrally limited slits, few (13\%) of females passed through 4-mm-high slits, and no individuals passed through 3-mm-high slits. Dorsoventral heights and pronotal widths of 930 individuals collected in Virginia were measured. Females were consistently larger, with pronota averaging $8.33 \mathrm{~mm}$ wide to the males' $7.47 \mathrm{~mm}$ and heights at the point of leg movement restriction averaging $4.03 \mathrm{~mm}$ to the males' $3.50 \mathrm{~mm}$. Based on experimental data and size data, we conclude that most $H$. halys individuals will be excluded by slits smaller than $3 \mathrm{~mm}$ and holes smaller than $7 \mathrm{~mm}$.
\end{abstract}

Key words: diapause, home invasion, brown marmorated stink bug, urban pest, building envelope

The brown marmorated stink bug, Halyomorpha halys (Stål), is a major agricultural pest and a significant domestic nuisance pest (Leskey et al. 2012, Rice et al. 2014, Leskey and Nielsen 2018). It has achieved its nuisance status because of its habit of using humanbuilt structures as refuges while in winter diapause. Every fall, these insects arrive at houses and sheds. They crawl around the exteriors while searching for entry points and may quickly enter when doors and windows are opened. In spring, they exit, triggered at least in part by the depletion of their fat bodies (Skillman et al. 2018). Throughout the colder months, individuals may exit their hiding places and move around indoors. This can be disturbing to human occupants, particularly in the cases of large infestations, as there can be tens of thousands of $H$. halys in a single house (Inkley 2012).

There are many methods to reduce pest pressure in buildings, and several have been applied for $H$. halys control. Indoors, a variety of light traps are commercially available, though a simple setup of a lamp over a pan of soapy water may be most effective (Aigner and Kuhar 2014). The application of pesticides and the use of traps on building exteriors have been shown to help reduce pressure (Watanabe et al. 1994). Pesticide-treated screens applied to windows may also be effective (Mooneyham et al. 2016). However, with the exception of treated screens, these methods are separate from the building, and only treated screens are related to exclusion.

The Asian ladybird beetle, Harmonia axyridis (Pallas) (Coleoptera: Coccinellidae), is a winter nuisance pest in similar ways to $H$. halys, and the sizes of these insects relevant to exclusion have been studied. Nalepa (2009) found that Ha. axyridis was unable to traverse 2-mm openings, but mostly passed through 3-mm openings. This information has found its way into some exclusion guidelines, which recommend $1.6-\mathrm{mm}$ (1/16 in) mesh installation over openings 
in houses that suffer ladybird beetle infestations (e.g., Layton 2014). In some places, similar screens can be used to stop $H$. halys ingress.

Exclusion methods apply to both new construction and existing buildings, although one must be careful to understand the origin and utility of openings, because some are necessary. Unintended or undesired openings in building envelopes often exist, serving as points of ingress for pests. These may occur because of the mistakes made during construction or because of moisture and temperature-related distortion of materials as buildings age. Intentional openings may be vital elements of ventilation systems. It is useful to understand the minimum size opening through which a pest can fit, so that pest control measures can be designed to exclude pests without obstructing ventilation and causing building failures. In this way, $H$. halys can be kept from entering through openings that are unnecessary and can also be kept from blocking those that are necessary.

There are two dimensions of $H$. halys likely to limit its passage through an opening. Although several previous studies have discussed dimensions of $H$. halys, they have not discussed dorsoventral dimensions or the ability of these insects to navigate through openings. The North American redescription of the species gave a length range of $12-17 \mathrm{~mm}$ and a width across humeral angles of 7-10 $\mathrm{mm}$ (Hoebeke and Carter 2003). Medal et al. (2013) gave the lengths of laboratory-reared adults ( $n=30$ for each sex) as $14.35 \mathrm{~mm}$ (range $12.8-15.5 \mathrm{~mm}$ ) for females and $11.97 \mathrm{~mm}$ (range 11.1-13.5 mm) for males. Pronotal widths have been reported in several studies of the nutritional statuses of Japanese $H$. halys populations (Funayama $2008,2012,2015)$. These studies indicated seasonal and annual fluctuations in sizes, but mean pronotal widths were all between 7 and $8 \mathrm{~mm}$ in males and between 8 and $9 \mathrm{~mm}$ in females, with SE extending beyond those ranges in very few cases. No published work presented the actual heights (dorsoventral dimensions) of $H$. halys. This is a difficult dimension to measure due to body and leg morphology and the lack of rigidity of the exoskeletons in this dimension compared with lateral or anterior-posterior dimensions. The flexibility of $H$. halys in this dimension can be observed by watching one walk on a surface, then lightly pressing down on it until the dorsal plane is forced to make light contact with the bottom surface. The legs become splayed, but observers will note that they are still able to move.

The study presented here seeks to further characterize the dorsoventral and pronotal dimensions of $H$. halys critical to its passage through building envelopes, and tests the species ability to traverse openings that limit access by these dimensions by encouraging them to pass through openings of decreasing size. The findings should inform design and maintenance decisions for appropriate treatment of openings in the building envelope to exclude pests while maintaining proper building performance.

\section{Materials and Methods}

\section{Insects}

Adult $H$. halys were collected from trees and the sides of buildings in Blacksburg, VA, during September and October. Insects were kept in mesh cages outdoors until use. Cages were shaded from sun and wind. Insects were provided branches and foliage of assorted plants, and provided with apples, carrots, and water-soaked cotton. No insects were reused in our assays.

\section{Apparatus and Procedure}

Test apparatus boxes were laser cut from 3.2-mm-thick hardboard. Pilot testing indicated that $H$. halys had no difficulty walking on this material, even on the undersides of lids. Pieces were designed to interlock. Assembled boxes were 200-mm cubes with removable lids. Outside edges were secured with blue masking tape and then completely blacked out with black electrical tape. Each box was only used once.

Two lid styles were used for the boxes. One was designed to limit exit by dorsoventral dimensions (height), and the other by lateral pronotal dimensions (width). Laterally limited exit lids each had four equal laser-cut circular holes (Fig. 1a). The multiple holes were provided to reduce the impact of competition for egress and blockage by insects that were too large to fit. The circular holes were 7, 8, 9, or $10 \mathrm{~mm}$ in diameter. Lids with dorsoventrally limited openings each had one laser-cut rectangular slit (Fig. 1b). Slits were $150 \mathrm{~mm}$ in length and 3, 4, or $5 \mathrm{~mm}$ wide. Boxes were empty except for test subjects, and all six inner surfaces were free of obstruction.

Before each trial, 30 male and 30 female $H$. halys were taken from the outdoor cages and chill-stunned in a refrigerator at $2^{\circ} \mathrm{C}$ for $30 \mathrm{~min}$. Insects were then placed in a box, the lid secured, and top edges blacked out with strips of black electrical tape. The box was then put into a mesh cage on top of a preheated DeLonghi (Treviso, Italy) SafeHeat TR0715L circulating oil space heater set to Medium-4 (Fig. 2). The apparatus was placed directly under an illuminated light bulb to help insects locate the exit. No temperature measurement device was used, but pilot tests indicated that

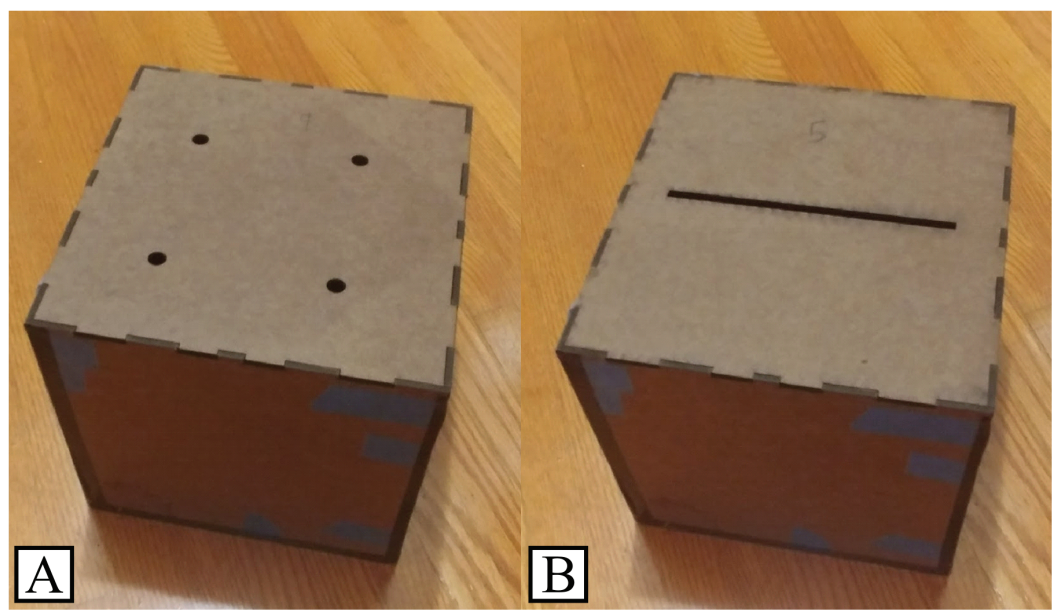

Fig. 1. (A) Laterally limited circular holes and (B) dorsoventrally limited rectangular slit. 
this heat provided sufficient stimulus for $H$. halys to exit the boxes, without mortality (B. D. Chambers, unpublished data). Other environmental conditions were similarly considered negligible due to the strong negative response of $H$. halys to the heat stimulus during pilot tests. After $5 \mathrm{~h}$, the boxes were moved into separate mesh cages to separate those insects that had exited from those that had not. The procedure was performed once $(n=60)$ for each of the seven treatments.

\section{Insect Dimension Measurement}

All escaping and nonescaping $H$. halys were measured with a digital caliper, as was as another group of insects from pilot tests. The pronota of $H$. halys are laterally rigid enough for easy and consistent measurement, but they are slightly dorsoventrally compressible. Therefore, dorsoventral height dimensions were taken at the point of compression at which the legs ceased to be able to freely move, confirmed by a light finger tap on the abdomen. Both experimental and pilot test measurements were included in the height and width summary statistics $(n=930)$. All 930 measurements included are from $H$. halys collected in late September and early October of 2015 in Blacksburg, VA. Insects were only measured after trials.

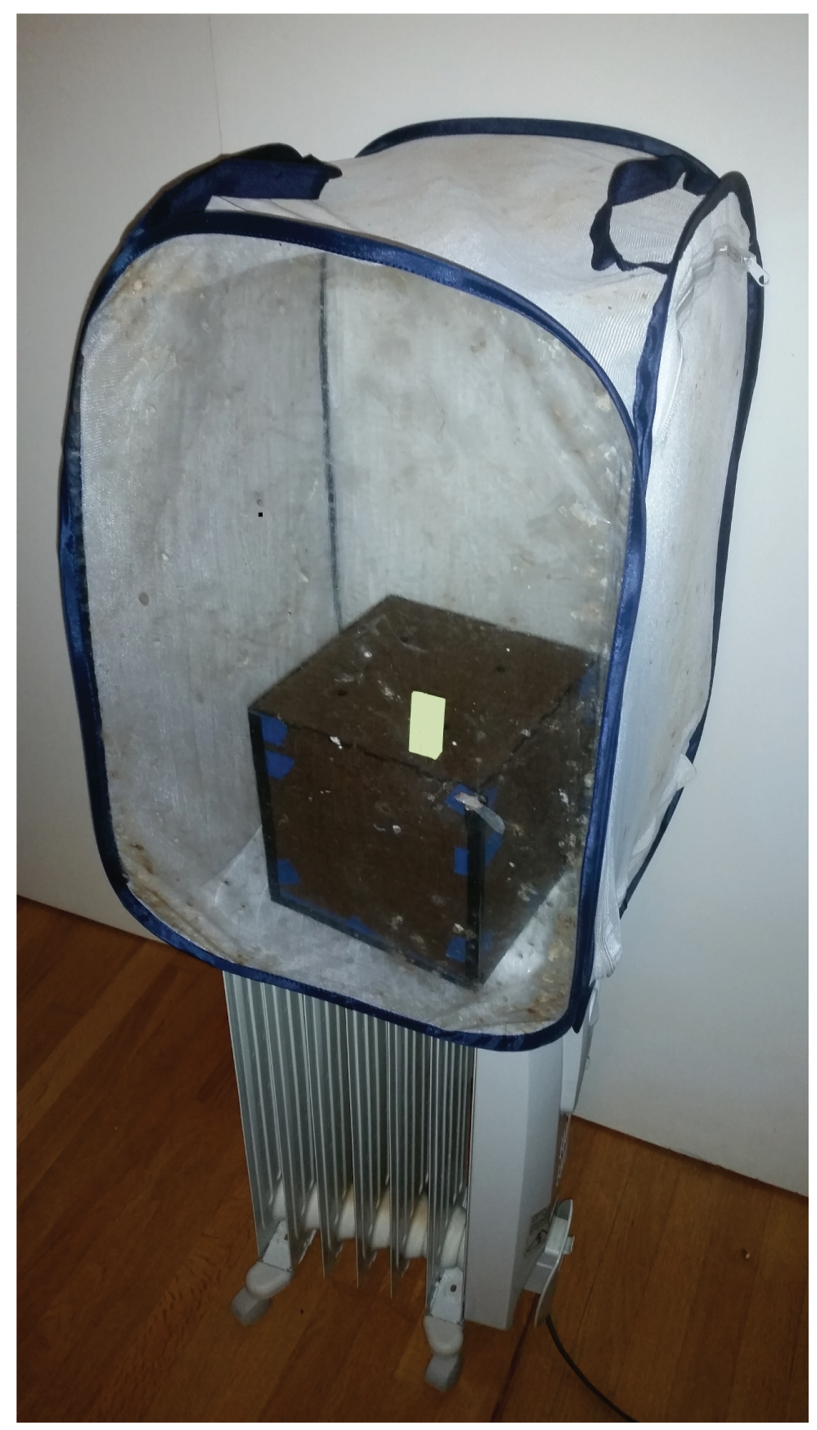

Fig. 2. Box in mesh cage atop a space heater.

\section{Statistical Analysis}

All analyses were performed with JMP Pro 13 (SAS Institute 2016). One-way analysis of variance (ANOVA) tests were conducted to compare means of pronotal and dorsoventral dimensions between sexes. Means of limiting dimensions for each sex and opening type were also analyzed using one-way ANOVA. Additional $t$-tests compared means of relevant dimensions between escaping and nonescaping insects for some opening sizes.

\section{Results}

The percentage of $H$. halys adults that passed through openings (escaped) was correlated with opening size, and more males escaped, consistent with their smaller size than females. Fewer insects passed through pronotally limited holes as hole size decreased (Fig. 3a), as well as through dorsoventrally limited slits (Fig. 3b). The $\leq 8-\mathrm{mm}$ holes and $\leq 4-\mathrm{mm}$ slits excluded most females. Only one male passed through the 7-mm-diameter holes, and one female through the $8-\mathrm{mm}$ holes. No insects passed 3-mm slits. Means of limiting dimensions for each sex across opening sizes were not significantly different for either opening type (Table 1). Openings approaching the size limit separated insects by their limiting dimensions. The pronotal dimensions of the males exiting the 8 -mm hole were significantly different from those remaining within the box (respective means 7.31, $7.56 \mathrm{~mm} ; t=-2.3 ; \mathrm{df}=29 ; P=0.0285$ ). Similarly, the dorsoventral dimensions of the females exiting the 4-mm slit were significantly different from those remaining within the box (respective means $3.78,4.09 \mathrm{~mm} ; t=-3.9 ; \mathrm{df}=29 ; P=0.0005)$.

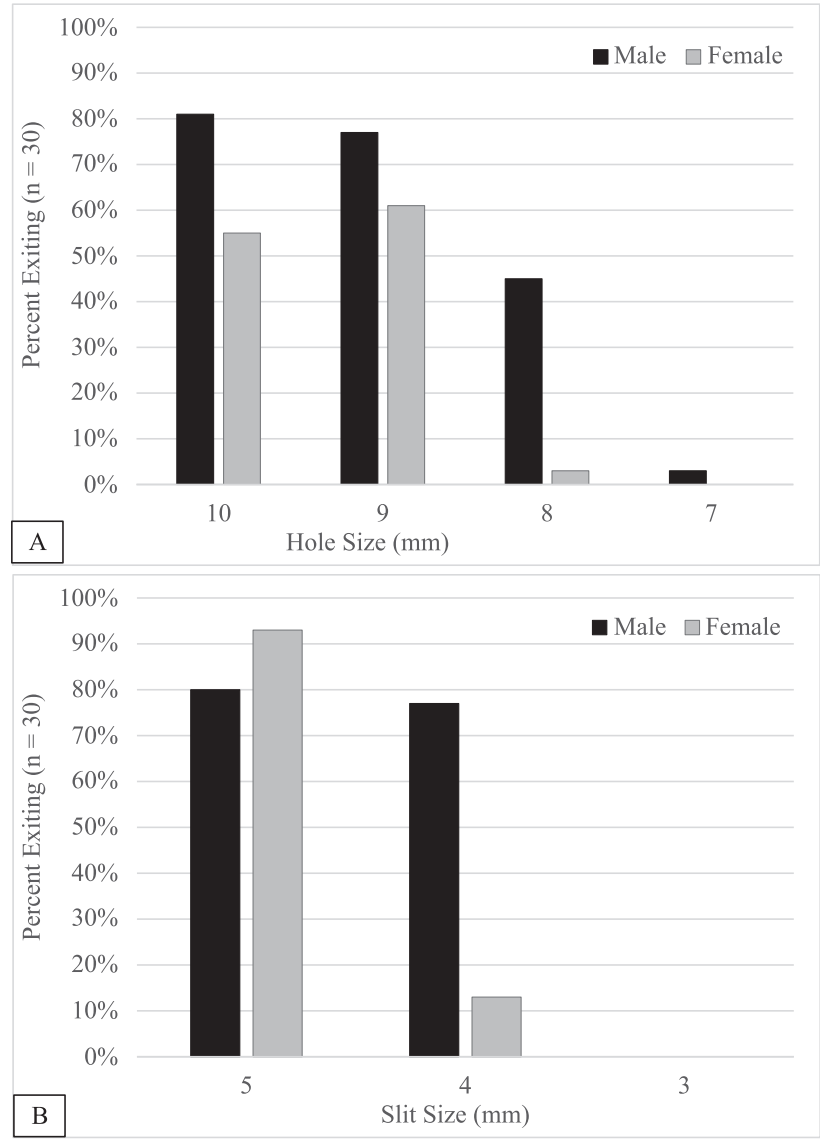

Fig. 3. Percentages of Halyomorpha halys adults escaping through each (A) pronotally limited hole and (B) dorsoventrally limited slit. 
Table 1. ANOVA results comparing means of limiting dimensions for each opening type

\begin{tabular}{|c|c|c|c|c|c|}
\hline Opening type & Dimension & Sex & $F$ & df & $P$ \\
\hline Hole & Pronotal & $\mathrm{F}$ & 0.0770 & 3,119 & 0.9723 \\
\hline Hole & Pronotal & M & 1.0155 & 3,121 & 0.3884 \\
\hline Slit & Dorsoventral & $\mathrm{F}$ & 0.2044 & 2,90 & 0.8155 \\
\hline Slit & Dorsoventral & M & 0.2868 & 2,89 & 0.7513 \\
\hline
\end{tabular}

Table 2. Dimension summaries from all adult Halyomorpha halys measurements

\begin{tabular}{lll}
\hline & Female & \multicolumn{1}{c}{ Male } \\
\hline $\begin{array}{l}\text { Number measured } \\
\text { Pronotal width }\end{array}$ & 465 & 465 \\
$\quad$ Average \pm SEM $(\mathrm{mm})$ & $8.33 \pm 0.02$ & $7.47 \pm 0.02$ \\
$\quad$ Range $(\mathrm{mm})$ & $7.04-9.43$ & $6.00-8.25$ \\
Dorsoventral height & & \\
$\quad$ Average \pm SEM $(\mathrm{mm})$ & $4.03 \pm 0.01$ & $3.50 \pm 0.01$ \\
$\quad$ Range $(\mathrm{mm})$ & $2.99-4.60$ & $2.41-4.00$ \\
\hline
\end{tabular}

For size evaluation, a total of 930 adult $H$. halys were measured, half male and half female (Table 2). Females measured an average of $8.33 \mathrm{~mm}$ wide across the pronotum and $4.03 \mathrm{~mm}$ high. Males averaged $7.47 \mathrm{~mm}$ wide and $3.50 \mathrm{~mm}$ high. The smallest dimensions were $6.00 \mathrm{~mm}$ wide at the pronotum and $2.41 \mathrm{~mm}$ high, both in males. Males were consistently smaller than females, both in terms of pronotal width (Fig. 4a) and dorsoventral height (Fig. 4b). Dorsoventral dimensions significantly differed between sexes $(F=1191.6 ; \mathrm{df}=1$, 928; $P<0.0001)$, as did pronotal widths $(F=1364.3 ; \mathrm{df}=1,928$; $P<0.0001)$.

\section{Discussion}

The average pronotal widths in our study are comparable to those measured by Funayama $(2008,2012,2015)$, with averages within $0.5 \mathrm{~mm}(6 \%)$. Though Funayama found that pronotal size varied from year to year, the variation was small enough that for exclusion related purposes, our findings may be generalized. We therefore conclude that few $H$. halys will be able to pass through rigid holes less than $7 \mathrm{~mm}$ wide and slits less than $3 \mathrm{~mm}$ high.

Rodent exclusion typically calls for steel mesh, 6.35-mm (sold as $1 / 4$ inch) grid hardware cloth (Geiger and Cox 2012). The diagonals of this grid are approximately $9 \mathrm{~mm}$, which may be sufficiently wide for most adult $H$. halys to pass through. However, the difficulty of walking on and passing through this material should be evaluated in future research. How tightly affixed the mesh is at the edges is also important, as $H$. halys have been seen attempting to crawl underneath in the homes of the authors.

The fact that a portion of $H$. halys were measured at shorter heights than the exclusion slits is not surprising, as heights were taken at the point at which they could no longer move their legs. To move into and through an opening, H. halys need room to maneuver. The softness and flexibility of materials as well as the configuration of the opening relative to the surfaces that $H$. halys move along may be important, but that is left to future research. However, in spite of the results, given that roughly $10 \%$ of the total measured males were less than $7 \mathrm{~mm}$ wide at the pronotum, it is possible that 7-mm limited openings will not provide perfect exclusion, unless other factors are at play. Similarly, a small number of males were less than $3 \mathrm{~mm}$ dorsoventrally, and thus, these 3-mm limiters may not

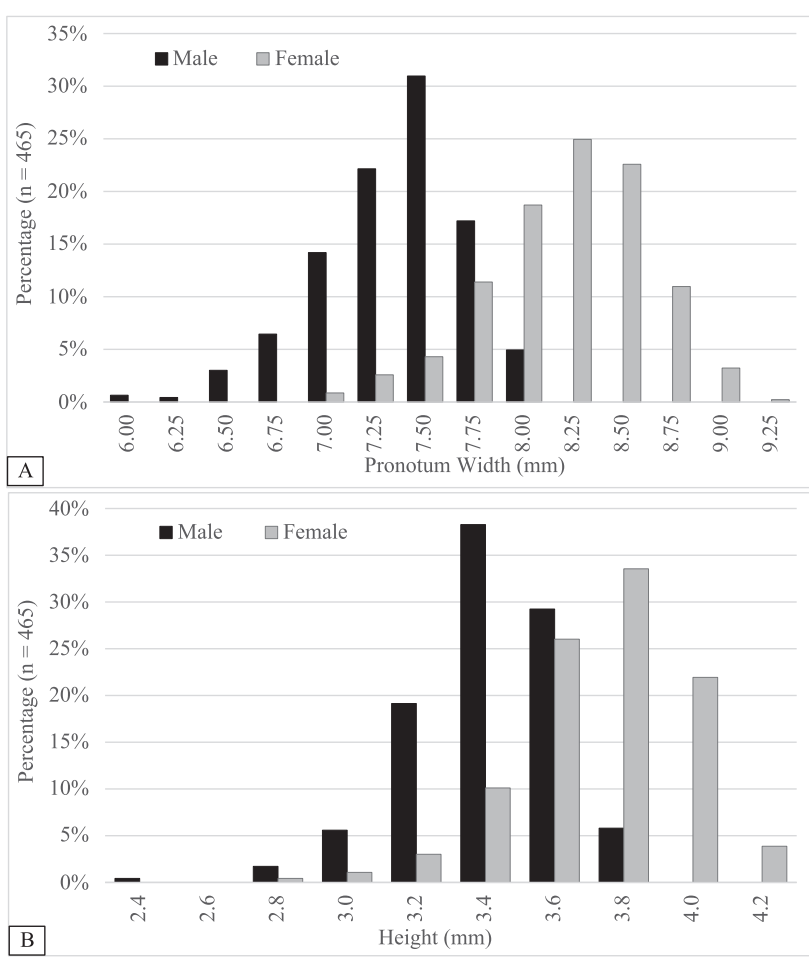

Fig. 4. Sizes of adult Halyomorpha halys by sex for (A) pronotal width and (B) height.

provide perfect exclusion. Although their small size may not make them less of a nuisance in homes, it is possible that these smaller individuals are underdeveloped and may not be relevant to population dynamics, as fat body has implications for both winter survival (Funayama 2012) and spring dispersal (Lee and Leskey 2015).

Pilot tests indicated that $H$. halys would try to escape heated boxes through any crack permitting light, and stay there for the duration of the test, even if it was too small. Blackout tape was used so that insects would only attempt to leave through the intended openings. It is unclear why $H$. halys did not abandon these cracks in favor of the larger ones through which they could fit. Some insects continued to stay in the corners after the blackout tape was installed. This suggests future research on interactions between heat and light in stress responses of $H$. halys.

The heat application, particularly after chill stunning, would be physiologically stressful for $H$. halys. However, although the heat was enough to drive most insects out of the box, it was not fatal. No insects in the trials presented here died, and only two died in pilot testing, both of which were small males. Temperatures were not recorded during the experiment, but they are assumed to be nonlethal for that duration. For perspective, a lethal high-temperature 4-h exposure test of $H$. halys reported $5.0 \pm 1.4 \%$ mortality at $35^{\circ} \mathrm{C}$ (Aigner and Kuhar 2016). Further exploration of heat-driven behaviors of $H$. halys may be useful for future experimental design. 
To learn more about how $H$. halys exited the openings, a GoPro camera (GoPro Inc., San Mateo, CA) recorded video of the box opening for several pilot iterations of the experiment. Halyomorpha halys moved through the openings sideways or diagonally, and typically walked forward to the edges of boxes before moving to cage walls. In one video, an individual crawled forward away from the slit, paused, and then backed up. Once it had moved its abdomen over the slit, it defecated. This was a small amount of fluid, but excretion has the potential to influence the dorsoventral compressibility of $H$. halys, so future research exploring excretion patterns during shelter seeking may be relevant. Although defecation also suggests the possibility of pheromonal action in the boxes, we consider any effects negligible due to the strength of response to the heat stimulus.

Results show that there are differences in the ability of males and females to pass through both types of opening. This is expected, given the results showing males as smaller than females in both measured dimensions. There are some morphological differences in males and females (Medal et al. 2013), but the influence of those on the ability of $H$. halys to physically navigate openings of different configurations and rigidity is left to future research.

These results provide dimensions to consider when treating structures for exclusion of $H$. halys. Any slit opening less than $3 \mathrm{~mm}$ is unlikely to permit the passage of many $H$. halys. Similarly, rigid holes or screen less than $7 \mathrm{~mm}$ wide should not suffer much entry. If other invading insects such as Ha. axyridis are also a concern, the suggested use of 1.6-mm mesh (typically sold in the United States as $1 / 16$ inch) is more than sufficient. In building systems, some materials are softer or more flexible and therefore could shift with temperature, moisture, or force of insect action, so this should be considered when designing or retrofitting for exclusion. Likewise, care needs to be taken that added screens do not reduce the effective area of a necessary opening lest moisture problems occur (Lstiburek 2006), particularly in buildings where vents are small and few in number.

\section{Acknowledgments}

Test apparatus construction was made possible by David Goldsmith and the Virginia Tech BuildLAB. Test subject collection assistance was provided by the Virginia Tech VIPR Lab. This research was supported in part by Virginia Tech BioBuild program and by USDA-NIFA SCRI awards \#2011-51181-30937 and \#2016-51181-25409.

\section{References Cited}

Aigner, J. D., and T. P. Kuhar. 2014. Using citizen scientists to evaluate light traps for catching brown marmorated stink bugs in homes in Virginia. J. Ext. 52: 4RIB5.

Aigner, J. D., and T. P. Kuhar. 2016. Lethal high temperature extremes of the brown marmorated stink bug (Hemiptera: Pentatomidae) and efficacy of commercial heat treatments for control in export shipping cargo. J. Agric. Urban Entomol. 32: 1-6.

Funayama, K. 2008. Seasonal fluctuations and physiological status of Halyomorpha halys (Stål) (Heteroptera: Pentatomidae) adults captured in traps baited with synthetic aggregation pheromone of Plautia crossota stali Scott (Heteroptera: Pentatomidae). Jpn. J. Appl. Entomol. Zool. 52: 69-75.

Funayama, K. 2012. Nutritional states of post-overwintering adults of the brown-marmorated stink bug, Halyomorpha halys (Stal) (Heteroptera: Pentatomidae). Jpn. J. Appl. Entomol. Zool. 56: 12-15.

Funayama, K. 2015. Nutritional status of overwintering adults of the brown marmorated stink bug, Halyomorpha halys (Hemiptera: Pentatomidae), is affected by cone counts of Japanese cedar, Cryptomeria japonica, in northern Japan. Appl. Entomol. Zool. 50: 117-121.

Geiger, C. A., and C. Cox. 2012. Pest prevention by design: authoritative guidelines for designing pests out of structures. San Francisco Department of the Environment, San Francisco, CA. https://sfenvironment.org/sites/default/files/fliers/files/final_ppbd_guidelines_12-5-12.pdf

Hoebeke, E. R., and M. E. Carter. 2003. Halyomorpha halys (Stål) (Heteroptera: Pentatomidae): a polyphagous plant pest from Asia newly detected in North America. Proc. Entomol. Soc. Wash. 105: 225-237.

Inkley, D. B. 2012. Characteristics of home invasion by the brown marmorated stink bug (Hemiptera: Pentatomidae). J. Entomol. Sci. 47: 125-130.

Layton, B. 2014. Physical exclusion: the best treatment for home-invading insect pests. Miss. State Univ. Bug-Wise Newsl. 7: 1-2.

Lee, D. H., and T. C. Leskey. 2015. Flight behavior of foraging and overwintering brown marmorated stink bug, Halyomorpha halys (Hemiptera: Pentatomidae). Bull. Entomol. Res. 105: 566-573.

Leskey, T. C., and A. L. Nielsen. 2018. Impact of the invasive brown marmorated stink bug in North America and Europe: history, biology, ecology, and management. Annu. Rev. Entomol. 63: 599-618.

Leskey, T. C., G. P. Dively, C. R. R. Hooks, M. J. Raupp, P. M. Shrewsbury, G. Krawczyk, P. W. Shearer, J. Whalen, C. Koplinka, E. Myers, et al. 2012. Pest status of the brown marmorated stink bug, Halyomorpha halys in the U.S. Outlooks Pest Manag. 23: 218-226.

Lstiburek, J. 2006. Understanding attic ventilation. ASHRAE J. 48: 36-45.

Medal, J., T. Smith, and A. Santa Cruz. 2013. Biology of the brown marmorated stink bug Halyomorpha halys. Fla. Entomol. 96: 1209-1212.

Mooneyham, K. L., J. Aigner John D., and T. P. Kuhar. 2016. Control of brown marmorated stink bug with insecticide-treated window screens, 2015. Arthropod Manag. Tests. 41: 1-1.

Nalepa, C. A. 2009. Harmonia axyridis (Coleoptera: Coccinellidae) in buildings: relationship between body height and crevice size allowing entry. J. Econ. Entomol. 100: 1633-1636.

Rice, K. B., C. J. Bergh, E. J. Bergmann, D. J. Biddinger, C. Dieckhoff, G. Dively, H. Fraser, T. Gariepy, G. Hamilton, T. Haye, et al. 2014. Biology, ecology, and management of brown marmorated stink bug (Hemiptera: Pentatomidae). J. Integr. Pest Manag. 5: 1-13.

SAS Institute. 2016. JMP Pro version 13.0.0. SAS Institute, Cary, NC.

Skillman, V. P., N. G. Wiman, and J. C. Lee. 2018. Nutrient declines in overwintering Halyomorpha halys populations. Entomol. Exp. Appl. 166: 778-789.

Watanabe, M., R. Arakawa, Y. Shinakawa, and T. Okazawa. 1994. Methods for preventing brown marmorated stinkbug infestation. Jpn. Soc. Med. Entomol. Zool. Anim. Heal. 45: 311-317. 